\title{
Target-depth estimation in active sonar: Cramer-Rao bounds for a bilinear sound-speed profile
}

\author{
Alexis Mours, ${ }^{\text {a) }}$ Cornel loana, and Jérôme I. Mars \\ Département DIS, Gipsa-Lab, 11 rue des Mathématiques, BP46, F-38402 Saint Martin d'Heres Cedex, France \\ Nicolas F. Josso and Yves Doisy \\ Département GSS, Thales Underwater Systems S.A.S., 525 Route des Dolines, BP 157, \\ 06903 Sophia Antipolis Cedex, France
}

(Received 15 March 2016; revised 28 July 2016; accepted 17 August 2016; published online 19 September 2016)

\begin{abstract}
This paper develops a localization method to estimate the depth of a target in the context of active sonar, at long ranges. The target depth is tactical information for both strategy and classification purposes. The Cramer-Rao lower bounds for the target position as range and depth are derived for a bilinear profile. The influence of sonar parameters on the standard deviations of the target range and depth are studied. A localization method based on ray back-propagation with a probabilistic approach is then investigated. Monte-Carlo simulations applied to a summer Mediterranean sound-speed profile are performed to evaluate the efficiency of the estimator. This method is finally validated on data in an experimental tank. (ㅇ 2016 Acoustical Society of America.
\end{abstract}

[http://dx.doi.org/10.1121/1.4962495]

[SED]

Pages: $1771-1782$

\section{INTRODUCTION}

In active sonar, the objectives are to detect, localize, and classify an underwater target. In the context of deep water, and particularly for a summer Mediterranean sound-speed profile, this paper focuses first on a new target-localization method that estimates the bearings, distance, and depth. The depth is estimated using vertical bearings and arrival-time estimation of a multipath environment. The target depth can then be used as a good feature for target classification or discrimination. This paper focuses secondly on new Cramer-Rao lower bounds (CRBs) of the target position, to quantify targetdepth errors. The CRBs are derived for a bilinear sound-speed profile (SSP) that can be an approximation for most real SSPs.

In the context of active sonar, horizontal bearings and distance are often used to localize targets. The depth may also be used as the key tactical information for strategy purposes, or as a good feature for target classification or discrimination. If an array has a vertical extent, then the elevation can be estimated and used as information to localize the target in terms of its depth and range. Two-dimensional (2-D) arrays as flank arrays, cylindrical arrays, and hull-mounted arrays use their vertical extent to estimate elevation angles. Even linear towed arrays can give some information about the elevation using the different conical bearings measured when multipath propagation arises.

Source localization can be seen as an inverse problem that can be solved with different model-based processing, such as matched-field processing, back-propagation and time-reversal techniques, and correlation processing. ${ }^{1}$ Matched-field processing techniques have often been used in the literature. These methods estimate the source position by

\footnotetext{
${ }^{a)}$ Electronic mail: alexis.mours@gipsa-lab.grenoble-inp.fr
}

matching the acoustic fields measured at an array of sensors with a replica field synthesized by a full-field acoustic model. An overview of the first methods was provided by Baggeroer. ${ }^{2}$ Matched-field methods are good source-location techniques when the environment parameters are known. However, acoustic ocean parameters vary with time and location, and matched-field processing is very sensitive to these parameters. Therefore, many studies have been developed to increase the robustness for the acoustic ocean parameter uncertainties. For example, the application of Bayesian inference to source localization problems was introduced by Richardson and Nolte: ${ }^{3}$ the uncertainties of the source and the environment parameters were used to infer the a posteriori probability of the source location. Dosso and Wilmut investigated different Bayesian approaches, using the source and environment parameter uncertainties on both simulated ${ }^{4}$ and $\mathrm{real}^{5}$ data. The acoustic focalisation ${ }^{6}$ and ray back-propagation $^{7-9}$ concepts are possible alternatives to matched-field processing, which has a huge computational load due to the large number of unknown parameters, including sound-speed profile parameters, array location, and bottom depth.

Concepts and methods relative to back-propagation in ocean acoustic inversion were well defined and discussed by Meyer and Hermand. ${ }^{1}$ The use of ray back-propagation to determine source location was first introduced by Collins and Kuperman, ${ }^{6}$ and then by Voltz and Lu. ${ }^{7}$ The timedomain ray back-propagation method ${ }^{7}$ exploits both the temporal and spatial characteristics of the multipath arrival structure at a receiving sensor array. The angles and relative arrival times are estimated and used to back-propagate the rays in a ray-trace program, to converge spatially on the source location. Then a new ray travel-time inversion scheme was developed by Lu to simultaneously estimate the source location, the array tilt, and the environmental parameters. ${ }^{8}$ The robustness of this approach has been tested through 
different parameter errors. ${ }^{9}$ This method gives efficient results on simulated and real data. However, all of these approaches are realized in a passive sonar context to localize the source.

In contrast to the passive sonar context, which needs an opportunity signal from the source, active sonar allows the detection and localization of a submerged target, that is silent or has a higher range. Moreover, active sonar has the advantage that it provides access to an estimate of the target distance instantaneously. In the literature of source/target localization, the passive sonar context is particularly seen, while there have been few studies that have talked about the active sonar context. An approach using depth dependence of relative delays and elevation angle spreads was investigated by Hickman and Krolik. ${ }^{10}$ Their method was applied with a horizontal array in shallow water, and it provided good results, although it needed several pings to converge to a good estimation of the target depth. Hjelmervik estimated the depth of a pipeline that was $4 \mathrm{~km}$ away using midfrequency active sonar data, ${ }^{11}$ although several pings were necessary to provide a depth probability density function. Management of combined rays, which are the rays that have a different path between the sonar and the target and between the target and the array, has also not been studied.

This paper is organized as follows: Sec. II describes the target localization problem and presents the CRBs for a bilinear SSP. The influence of some sonar parameters is also studied for the CRBs. Section III presents a new target-depth estimation method that is based on ray back-propagation with a probabilistic approach, and combined path management. Section IV discusses the results of two Monte-Carlo simulations. Section V presents the results of the targetdepth estimation on the experimental data recorded in a reduced-scale water tank. The conclusions follow in Sec. VI.

\section{CRAMER-RAO LOWER BOUNDS FOR BILINEAR SOUND-SPEED PROFILES}

This section presents the target localization problem, and investigates the new CRBs of the target position, especially for the target depth, in a bilinear SSP. The bilinear SSP was chosen because most real SSPs can be approximated by bilinear SSPs. Part of these CRBs have already been proposed by Blanc-Benon and Jauffret in a target motion analysis context; ${ }^{12}$ they derived the CRBs of a target position as the range, depth, azimuth, and target speed vector of a moving target using horizontal bearings and multipath time-delay estimations. In the present paper, the CRBs of a target position are derived for a single sonar ping using the vertical bearings and arrival-time estimations.

\section{A. Problem statement}

Consider a sonar composed of an acoustic source and a receiver that are co-located at $\left(R=0, Z=z_{0}\right)$, and a target that is positioned at $(R=r, Z=z)$ in a water column. Figure 1 illustrates the context of an active sonar and a submerged target. The bilinear SSP is described by three pairs of two inputs (i.e., the sound speed, and the depth): $\left[c_{1} z_{1}\right],\left[c_{2} z_{2}\right]$, and $\left[c_{3} z_{3}\right] . Z=z_{1}$ represents the horizontal surface, and
$Z=z_{3}$ represents the bottom surface. $c_{2}$ is the minimum value of the SSP at the depth $Z=z_{2}$. The $Z$ axis is taken as positive downwards from the surface. The sonar and the target are linked by eigenrays that represent acoustic paths taken by the sound wave to go from a point A to a point B. $\varphi$ is the elevation angle between the ray and the horizontal axis at the sonar position, and $\Phi$ is the elevation angle between the ray and the horizontal axis at the target position. The ray angles are defined as positive clockwise from the horizontal.

We assume that one sonar ping was emitted from a sonar, reflected by a target, and then finally recorded by an array. We consider that the target is in the azimuth plan of the sonar and that the measurements are defined in the sonar reference frame. After the signal-processing steps (i.e., beamforming, matched filter, normalization), there are $N$ detected wavefronts. A wavefront can be characterized by three measurements: the elevation $\varphi$, the wave travel time $\tau$, and the Doppler compression coefficient $\eta$. In active sonar, path combinations can occur, as acoustic waves can travel in another path after their reflection on the target. To simplify the problem for the CRBs, we assume that the one-way return travel time $T$ (i.e., time delay from target to array) is estimated from the measurement of the two-way travel time $\tau$ (i.e., time delay sonar to target, and then to array) for $L$ detections. $L$ corresponds to the number of different elevation in the detection of a single ping, and is often equal to 2 or 3 in this paper. So only the one-way return travel time $T$ will be used in the derivation of the CRBs. The target is defined by the vector of random variables $\boldsymbol{X}=[r, z]^{t}$. The sonar model is defined by the vector

$$
M=\left[\begin{array}{lll}
\boldsymbol{\varphi}^{t} & \boldsymbol{T}^{t} & \boldsymbol{\eta}^{t}
\end{array}\right]^{t},
$$

where $\boldsymbol{\varphi}=\left[\varphi_{1}, \ldots, \varphi_{L}\right]^{t}$ are the elevation angles, $\boldsymbol{T}=\left[T_{1}\right.$, $\left.\ldots, T_{L}\right]^{t}$ are the one-way return travel times, $\boldsymbol{\eta}=\left[\eta_{1}, \ldots, \eta_{L}\right]^{t}$ are the Doppler compression coefficients, $L(\leq N)$ is the number of detections used, and ${ }^{t}$ is the transpose operator. The measurements are defined as follows:

$$
M^{(m)}=M+\boldsymbol{\delta},
$$

where $\boldsymbol{\delta}$ is the noise vector and can be decomposed as follows:

$$
\boldsymbol{\delta}=\left[\begin{array}{lll}
\boldsymbol{\delta} \varphi^{t} & \boldsymbol{\delta} \boldsymbol{T}^{t} & \boldsymbol{\delta} \boldsymbol{\eta}^{t}
\end{array}\right]^{t},
$$

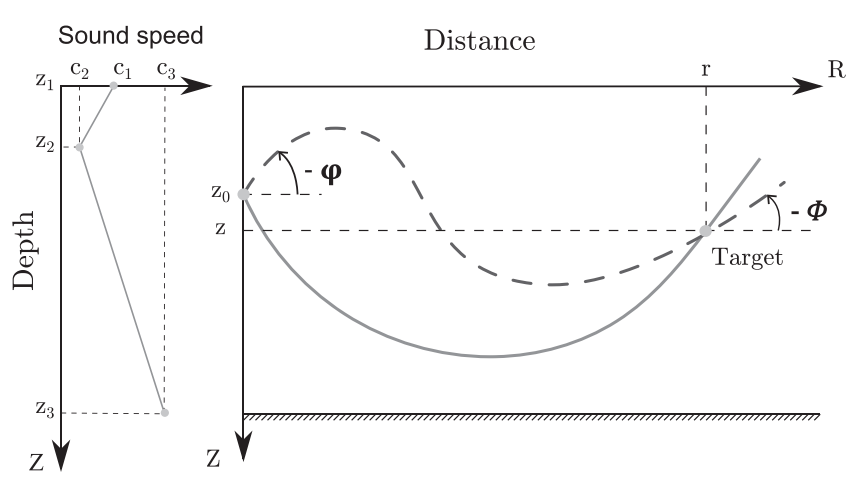

FIG. 1. Geometric conventions for the bilinear sound-speed profile, for sonar and target position, and for eigenrays. Example with two eigenrays. 
where $\delta \boldsymbol{\varphi}=\left[\delta \varphi_{1}, \ldots, \delta \varphi_{L}\right]^{t}, \delta \boldsymbol{T}=\left[\delta T_{1}, \ldots, \delta T_{L}\right]^{t}$, and $\delta \eta$ $=\left[\delta \eta_{1}, \ldots, \delta \eta_{L}\right]^{t}$ are vectors of independent zero-mean Gaussian noise with known standard deviations $\sigma_{\varphi}, \sigma_{T}$, and $\sigma_{\eta}$, respectively. The full covariance matrix is defined as follows:

$$
\Sigma=\operatorname{diag}\left(\sigma_{\varphi_{1}}^{2}, \ldots, \sigma_{\varphi_{L}}^{2}, \sigma_{T_{1}}^{2}, \ldots, \sigma_{T_{L}}^{2}, \sigma_{\eta_{1}}^{2}, \ldots, \sigma_{\eta_{L}}^{2}\right) .
$$

The independence assumption between the Doppler coefficient and delay measurements is true only for certain waveforms: pulse train frequency modulation and binary phase shift keying. This is not true for the Linear Frequency Modulation (LFM) which has a delay-Doppler coupling. This approximation for the LFM waveform may impact the Cramer-Rao bounds results when the Doppler coefficient and the delay are used together, that means only in the study of the influence of the target speed vector on the CRBs.

\section{B. The Cramer-Rao bounds derivation}

The CRBs give information on the uncertainties of the estimates with regards to the input parameters. For an unbiased estimator $\hat{X}$, the CRBs are defined as the inverse of the Fisher information matrix, which is denoted as $F(X)$ :

$$
\operatorname{var}(\hat{X}-X)=F(X)^{-1} .
$$

With a Gaussian noise model, the Fisher information matrix verifies as ${ }^{13,14}$

$$
F=\left(\frac{\partial M(X)}{\partial X}\right)^{t} \Sigma^{-1}\left(\frac{\partial M(X)}{\partial X}\right)
$$

where $M(X)$ is the noiseless measurement vector, and $J$ $=\partial M(X) / \partial X$ is the Jacobian matrix. Here the Jacobian $J$ is a $3 L \times 2$ matrix, where $L$ is the number of detections used for the same target.

$$
J=\left[\begin{array}{ll}
\frac{\partial \boldsymbol{\varphi}}{\partial r} & \frac{\partial \boldsymbol{\varphi}}{\partial z} \\
\frac{\partial \boldsymbol{T}}{\partial r} & \frac{\partial \boldsymbol{T}}{\partial z} \\
\frac{\partial \boldsymbol{\eta}}{\partial r} & \frac{\partial \boldsymbol{\eta}}{\partial z}
\end{array}\right] .
$$

The two partial derivatives, $\partial \varphi / \partial r$ and $\partial \varphi / \partial z$, represent the inverse of the ray angular divergences for the range and depth, and these are computed using a ray-propagation simulator we developed. This simulator can directly provide these ray angular divergences for a target position. For SSPs with multiple constant gradients, the ray propagation from the source to the target position was realized by computation of successive ray arches. ${ }^{15}$ The next two partial derivatives, $\partial T / \partial r$ and $\partial T / \partial z$, are derived analytically from the equations system that connects the one-way travel time (i.e., sonar target, target array) to the target parameters in the bilinear SSP case, which was introduced by Baer and Jacobson, ${ }^{16}$ and is given as

$$
\begin{aligned}
& r=G(\varphi, z), \\
& T=H(\varphi, z),
\end{aligned}
$$

where functions $G$ and $H$ are as expressed in Appendix A, $r$ is the horizontal range, $T$ is the one-way return travel time, $\varphi$ is the elevation angle at the sonar, and $z$ is the target depth. The elevation angle $\varphi$ is implicitly defined by Eqs. (8) and (9). Recalling the expressions of $G$ in Eq. (A1) and $H$ in Eq. (A2), Blanc-Benon derived analytically the partial derivatives of the one-way travel time to the target parameters using the theorem of implicit function ${ }^{12}$ as

$$
\begin{aligned}
& \frac{\partial T}{\partial r}=\frac{\partial H}{\partial \varphi} / \frac{\partial G}{\partial \varphi} \\
& \frac{\partial T}{\partial z}=-\frac{\partial H}{\partial \varphi} \frac{\partial G}{\partial z} / \frac{\partial G}{\partial \varphi}+\frac{\partial H}{\partial z}
\end{aligned}
$$

The four derivatives, $\partial H / \partial \varphi, \partial H / \partial z, \partial G / \partial \varphi$, and $\partial G / \partial z$, are derived analytically and are expressed in Appendix B. The last partial derivatives of Eq. (7), $\partial \eta / \partial r$ and $\partial \eta / \partial z$, are new and concern the small variations of the Doppler compression coefficient with respect to target position. In the active sonar and multipath environment, the Doppler compression coefficient $\eta$ can be written as follows: ${ }^{17,18}$

$$
\eta \approx 1-\frac{V_{i}}{c(z)}-\frac{V_{r}}{c(z)}
$$

where $c(z)$ is the sound speed at the target depth $z$, and $V_{i}$ (respectively, $V_{r}$ ) is the target radial speed of the incident eigenray (respectively, the reflected eigenray). The target radial speeds of the incident and reflected rays are projections of the target speed vector on the incident and reflected eigenrays, as shown in Fig. 2, and they can be expressed as follows:

$$
\begin{aligned}
& V_{i}=\vec{V}_{t} \cdot \vec{u}_{i}=\left\|\vec{V}_{t}\right\| \cos \left(\Phi_{t}-\Phi_{i}\right), \\
& V_{r}=\vec{V}_{t} \cdot \vec{u}_{r}=\left\|\vec{V}_{t}\right\| \cos \left(\Phi_{t}-\Phi_{r}\right),
\end{aligned}
$$

where $\vec{V}_{t}$ is the target-speed vector, $\Phi_{t}$ is the angle between the target-speed vector and the horizontal axis, $\Phi_{i}$ and $\Phi_{r}$ are

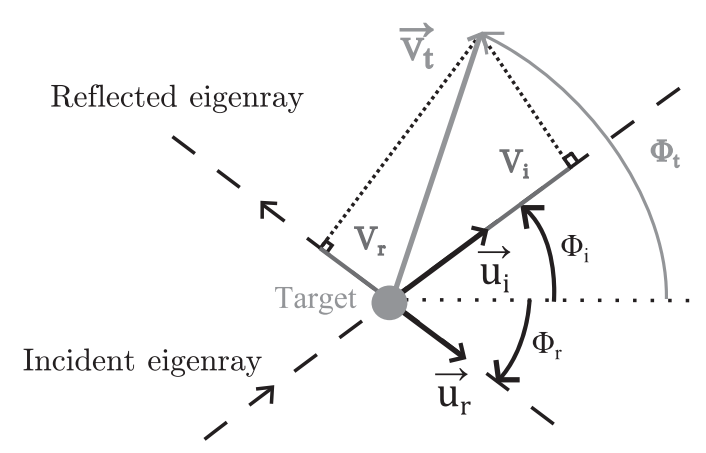

FIG. 2. Projection of the target-speed vector on an eigenray. $V_{i}$ and $V_{r}$ are the projection of the target-speed vector on incident and reflected eigenray. $\overrightarrow{V_{\text {proj }}}$ and $\Phi$ are the speed vector viewed by an eigenray and the elevation angle of the eigenray at the target, respectively. 
the incident and reflected eigenray angles, respectively, $\overrightarrow{u_{i}}$ and $\overrightarrow{u_{r}}$ are the unity vectors of the incident and reflected eigenray, respectively, and $\eta$ is the Doppler compression coefficient. To simplify the CRB analysis, the reflected and incident rays are assumed to be the same: $\Phi=\Phi_{i}=\Phi$. This hypothesis is strong, but it allows an easier notation and analysis. The partial derivative of $\eta$ relative to $r$ is expressed as follows:

$$
\frac{\partial \eta}{\partial r}=\frac{\partial \Phi}{\partial r} \frac{2\left\|\overrightarrow{V_{t}}\right\|}{c(z)} \sin \left(\Phi_{t}-\Phi\right) .
$$

By deriving the Snell-Descartes law relative to $r$, we obtain

$$
\begin{aligned}
& \frac{\cos \varphi}{c\left(z_{0}\right)}=\frac{\cos \Phi}{c(z)} \\
& -\frac{\partial \varphi}{\partial r} \frac{\sin \varphi}{c\left(z_{0}\right)}=-\frac{\partial \Phi}{\partial r} \frac{\sin \Phi}{c(z)} .
\end{aligned}
$$

Finally Eq. (15) becomes

$$
\frac{\partial \eta}{\partial r}=\frac{\partial \varphi}{\partial r} \frac{2\left\|\vec{V}_{t}\right\| \sin \varphi}{c\left(z_{0}\right) \sin \Phi} \sin \left(\Phi_{t}-\Phi\right) .
$$

The partial derivative $\partial \eta / \partial z$ can be derived using Eq. (A3), and can be expressed as follows:

$$
\begin{aligned}
\frac{\partial \eta}{\partial z}= & 2\left\|\vec{V}_{t}\right\|\left[\frac{\partial \varphi}{\partial z} \frac{\sin \varphi \sin \left(\Phi_{t}-\Phi\right)}{c\left(z_{0}\right) \sin \Phi}\right. \\
& \left.+\left(B g_{1}-D g_{2}\right) \frac{\cos \left(\Phi_{t}-\Phi\right)}{c^{2}(z)}\right] .
\end{aligned}
$$

The Doppler compression coefficient and these two partial derivatives vary according to two unknown parameters: the magnitude, and the angle of the target-speed vector. If these target-speed-vector parameters are known and the ray directions are orthogonal to the target movement, the Doppler derivative inclusion would probably decrease the variance of the target-position estimate. The Fisher information matrix and CRBs are finally computed for a given target position, and can be expressed as follows: ${ }^{13}$

$$
\mathrm{CRB}=\operatorname{var}(\hat{X}-X)=\left[\begin{array}{cc}
\sigma_{r}^{2} & \operatorname{cov}(r, z) \\
\operatorname{cov}(z, r) & \sigma_{z}^{2}
\end{array}\right],
$$

where $\sigma_{z}$ and $\sigma_{r}$ are the target-depth and target-range standard deviation bounds, respectively.

Figure 3 illustrates the geometric interpretation of the CRBs for two rays. Case 0 shows a reference frame of two ellipses with the semi-minor axis and semi-major axis represented by $\sigma_{T}$ and $f\left(\sigma_{\varphi}\right)$, respectively, where $f$ is the propagation function. Indeed, the 2-D Gaussian distribution in time and elevation angle is warped by the propagation channel, to give an ellipse in the range-depth plane. The product of the ellipses produces the uncertainty area shown in gray in Fig. 3. This area is characterized by the width $2 \sigma_{r}$ and the height $2 \sigma_{z}$. Case 1 indicates that a modification of the angle between the two ellipses changes the uncertainty area and thus the target-depth standard deviation bound $\sigma_{z}$. The angular diversity between the elevation angle measurements should be maximum, because an angular diversity at the receiver is likely to result in an angular diversity at the target, and therefore an improved target-depth standard deviation bound. Case 2 and case 3 show examples of the influence of the parameter standard deviations $\sigma_{T}$ and $\sigma_{\varphi}$, respectively. Case 2 reveals in this configuration that the expansion of $\sigma_{\varphi}$ has no influence on $\sigma_{z}$ and $\sigma_{r}$, and case 3 reveals that the expansion of $\sigma_{T}$ increases $\sigma_{z}$ and $\sigma_{r}$.

\section{Parameter influence on the standard deviation bounds}

The influence of the target position, frequency band, elevation-angle standard deviation, and target-speed vector on the target-depth and target-range standard deviation bounds are studied here. If the target sonar position and sonar parameters are fixed, and the SSP is known, the standard deviation bounds for the target position can be computed. We propose a realistic active sonar scenario with the following parameters:

(1) The bilinear SSP that corresponds to a summer Mediterranean SSP:

$$
\begin{aligned}
& \left(c_{1}, z_{1}\right)=(0 \mathrm{~m}, 1531 \mathrm{~m} / \mathrm{s}) \\
& \left(c_{2}, z_{2}\right)=(100 \mathrm{~m}, 1507 \mathrm{~m} / \mathrm{s}) \\
& \left(c_{3}, z_{3}\right)=(2500 \mathrm{~m}, 1546 \mathrm{~m} / \mathrm{s})
\end{aligned}
$$

(2) The sonar depth as $z_{0}=200 \mathrm{~m}$.

(3) The sonar code used is a LFM signal with a time duration $T_{e}=2 \mathrm{~s}$, a frequency band $B=500 \mathrm{~Hz}$, and a center frequency $f_{0}=5 \mathrm{kHz}$. These parameters give a time and speed resolution at $-3 \mathrm{~dB}$ as follows: ${ }^{18}$

$$
\begin{aligned}
& 2 \tau_{-3 \mathrm{~dB}}=2 T_{-3 \mathrm{~dB}}=2 \times \frac{0.44}{B}=1.8 \mathrm{~ms}, \\
& 2 V_{-3 \mathrm{~dB}}=2 \times \frac{c}{2} \times \frac{0.44}{T_{e} f_{0}}=0.066 \mathrm{~m} / \mathrm{s} .
\end{aligned}
$$

(4) The height of the array is $H=1 \mathrm{~m}$. The elevation angle resolution at $-3 \mathrm{~dB}$ in the broadside of the array is ${ }^{15}$

$$
2 \varphi_{-3 \mathrm{~dB}}=\frac{50 c}{f_{0} H}=15 \mathrm{deg} .
$$

The target is assumed to be in the broadside of the array in order to simplify the analysis. The elevation angle resolution is usually dependent of the array and the target azimuth. Given a specific array, this could be included in the analysis.

(5) The target at $r=18 \mathrm{~km}$ and $z=400 \mathrm{~m}$ has a speed of $V=0 \mathrm{~m} / \mathrm{s}$.

For a point-like target, the standard deviations of $T, V$, and $\varphi$ can be expressed for large SNRs as follows: ${ }^{15}$

$$
\sigma_{T_{i}}=\frac{T_{-3 \mathrm{~dB}}}{\sqrt{\mathrm{SNR}_{i}}}
$$



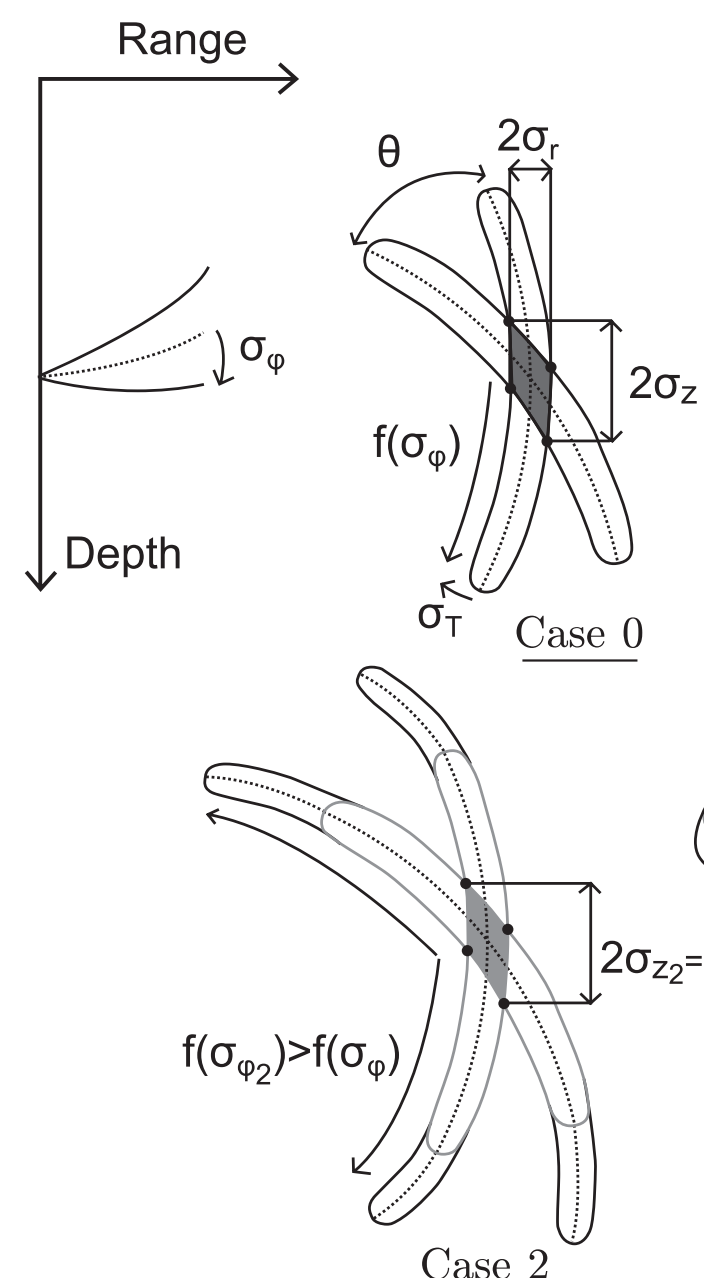

Case 2

$$
\begin{aligned}
& \sigma_{V_{i}}=\frac{V_{-3 \mathrm{~dB}}}{\sqrt{\mathrm{SNR}_{i}}}, \\
& \sigma_{\varphi_{i}}=\frac{\varphi_{-3 \mathrm{~dB}}}{\sqrt{\mathrm{SNR}_{i}}} \text { for } i=1, \ldots, L,
\end{aligned}
$$

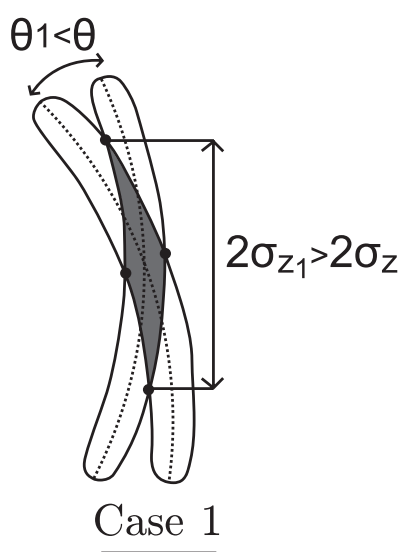

FIG. 3. Geometric interpretation of the CRBs with an example for two rays. Case 0: reference frame for two ellipses. Case 1: Influence of the angle $\theta$ between the two arcs of the circle. Case 2: Influence of the expansion of $\sigma_{\varphi}$, and therefore $f\left(\sigma_{\varphi}\right)$, with $f$ as the propagation function. Case 3: Influence of the expansion of $\sigma_{T}$. where $\mathrm{SNR}_{i}$ is the $\mathrm{SNR}$ of the detection $i$ at the array output in the linear scale. For a false alarm probability of $10^{-4}$ and a detection probability of $90 \%$, a detection will be true if the SNR is above approximately $15 \mathrm{~dB} .{ }^{19}$ In the present paper, the SNR will be set to $15 \mathrm{~dB}$ for all true detections, and the time delay, speed, and elevation standard deviations are therefore $230 \mu \mathrm{s}(35 \mathrm{~cm}), 1.1 \mathrm{~mm} / \mathrm{s}$, and $1.93 \mathrm{deg}$, respectively.

Figure 4 shows the standard deviation bound of the target depth as a function of the target range and depth according to Eq. (5) for two rays. The results indicate that the target-depth standard deviation bound decreases generally with the range. The sound propagation function in the water column is complex due to the bilinear form of the SSP. In certain areas, this propagation function gives lower or higher angular deviations for rays, and consequently a complex map of the target-depth standard deviation bound. It should be noted that areas around caustics, where the ray divergence tends to zero, give a satisfying target-depth standard deviation bound. The area from 20 to $40 \mathrm{~km}$ in range, and from 0 to $300 \mathrm{~m}$ in depth, shows a high depth uncertainty and it will be difficult to have a good target-position estimate there. These results can vary for different environments and geometric parameters [i.e., SSP, sonar depth, number of detections used $(L)]$.

The frequency band and the height of the array are parameters that can be controlled, and so their influence is studied here. The conclusions reached next are only valuable for the target position stated previously, but the methodology remains valid for any case. Figure 5 shows the plots of the target-depth and target-range standard deviation bounds, $\sigma_{z}$ and $\sigma_{r}$, respectively, as a function of the frequency band, $B$, and the number of detections used, $L$. It is clear that the number of rays used for the localization is a significant parameter to improve the standard deviations bounds. Low values of standard deviations bounds are not realistic, because errors

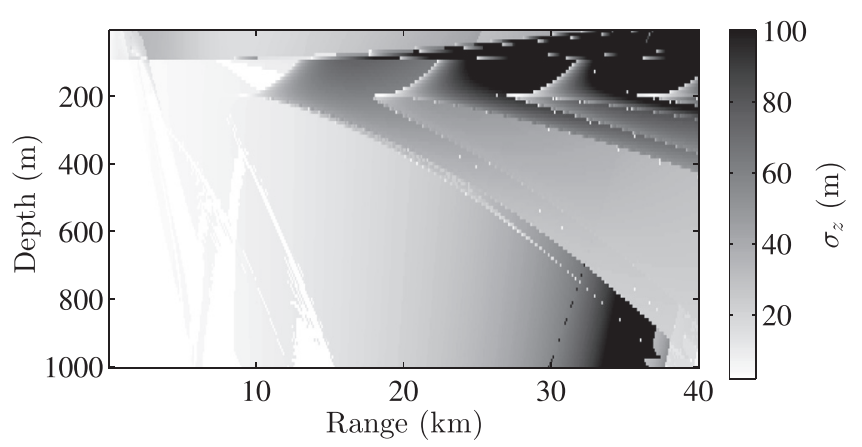

FIG. 4. Map of the target-depth standard deviation bound, $\sigma_{z}$. The color map represents the level of the standard deviation bound in meters. The example shown is for two rays. 

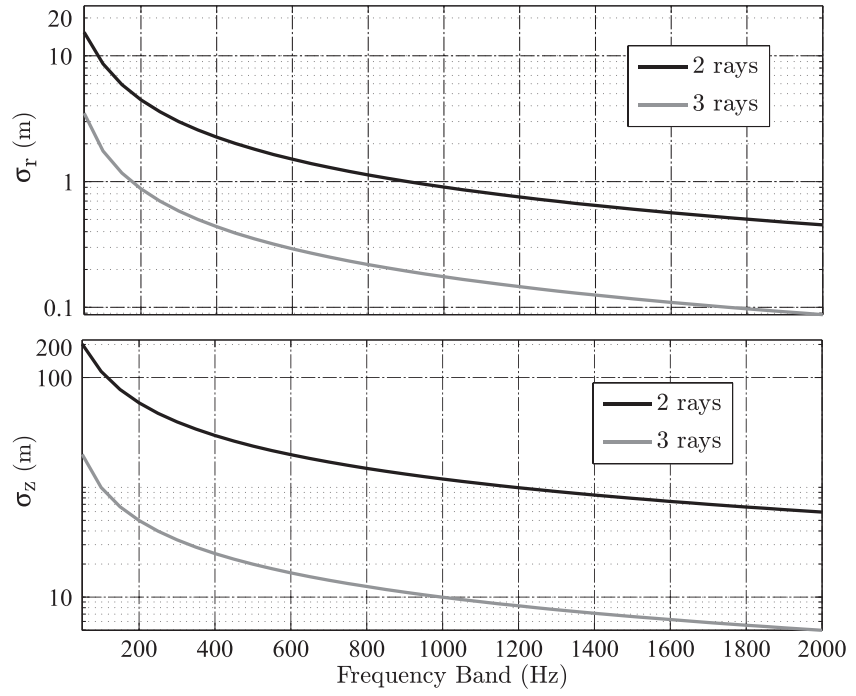

FIG. 5. (a) Plot of the target-range standard deviation bound $\sigma_{r}$ in logarithmic scale as a function of the frequency band $B$ for two and three rays. (b) Plot of the target-depth standard deviation bound $\sigma_{z}$ as a function of the frequency band $B$ for two and three rays.

due to ambient noise can become preponderant. Figure 6 shows plots of the target-depth and target-range standard deviation bounds, $\sigma_{r}$ and $\sigma_{z}$, respectively, as a function of the elevation-angle standard deviation, $\sigma_{\varphi}$. The results indicate that the target-depth standard deviation bound increases as the elevation-angle standard deviation increases (equivalent to a smaller array or lower SNR) for two rays. The improvements in the target-depth and target-range standard deviation bounds are still present as the number of rays increases. The convergence of the curves for two and three rays toward the asymptote is rapidly reached and can be explained by case 2 of Fig. 3. The intersection angle between the ellipses and the time standard deviation (equivalent to the band) are the predominant variables against the elevation standard deviation to modify the uncertainty area.

Figure 7 shows the polar plots of the target-depth and target-range standard deviation bounds, $\sigma_{r}$ and $\sigma_{z}$, respectively, as a function of the angle of the target-speed vector, $\Phi_{t}$, for three different target speeds: 0,10 , and $20 \mathrm{~m} / \mathrm{s}$. This reveals that $\sigma_{r}$ and $\sigma_{z}$ decrease when the target speed is greater than zero and when the angle of the target-speed

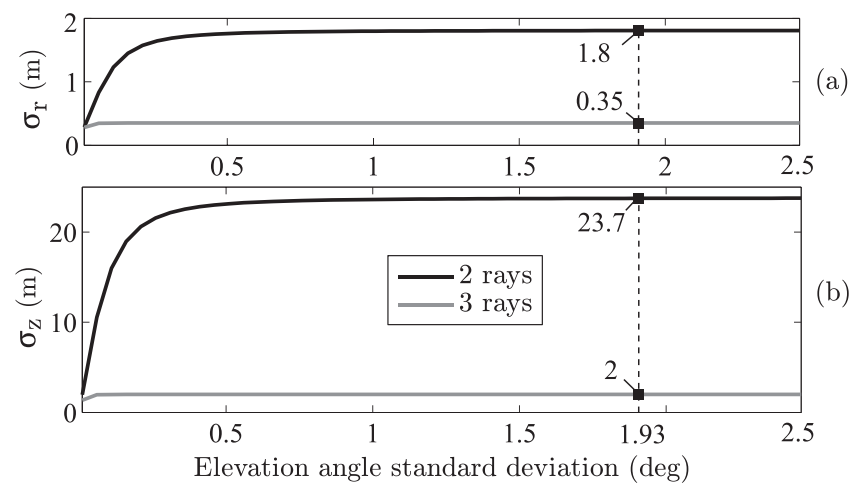

FIG. 6. (a) Plot of the target-range standard deviation bound $\sigma_{r}$ as a function of the elevation-angle standard deviation $\sigma_{\varphi}$ for two and three rays. (b) Plot of the target-depth standard deviation bound $\sigma_{z}$ as a function of the elevation-angle standard deviation $\sigma_{\varphi}$ for two and three rays. vector approaches $\pm 90 \mathrm{deg}$, which is orthogonal to the rays. This improvement in the standard deviation bounds becomes less important if the number of rays used rises. The use of the Doppler compression coefficient and the CRB results are valid assuming that the target-speed vector is known, which is not necessarily the case. The CRB results for the Doppler suggest that the Doppler compression coefficient can provide information on the target location if we know the targetspeed vector. We assume in the next part of the paper that the target-speed vector is unknown, so that the Doppler compression coefficients were not used.

This CRB study gives quantitative values for the standard deviation bounds and exposes the influence of the frequency band, the height of the array, the target location, and the target-speed vector on these bounds.

\section{TARGET LOCALIZATION}

Section II suggested that estimation of the target depth is possible with low uncertainty for many target locations. This section presents a localization method that is similar to the one proposed by Hjelmervik. ${ }^{11}$ This is based on ray back-propagation with a probabilistic approach, where elevations and time delays of detections are used as input variables. Measurement uncertainties due to finite arrays, emitted waveforms, and ambient noise, are included in the localization method. This estimator was also compared with the previous CRB results.

\section{A. Semi-active localization}

Our target localization algorithm is based on rays and back-propagation of the uncertainties. The principle is to propagate the measurement uncertainties of all of the detections from the array position to the target in a propagation simulator, and to compute a target-position probability density function. The first hypotheses of the problem are stated here.

(1) The target is assumed to be a single highlight spot (point target) model.

(2) The position of the moving target is the same for each acoustic path.

(3) The one-way return travel times of the $L$ detections used are estimated.

(4) The sound-speed profile at the array is known.

The first hypothesis considers a point-target model to assure that the eigenrays between the sonar and the target are similar to those between the target and the array. If the target moves during the wave propagation, the target position will be different for each acoustic propagation path. The second hypothesis therefore simplifies the problem of a moving target by considering it as a fixed target with Doppler. The consequences are the same as those for the first hypothesis. The third hypothesis implies that there are at least $L$ detections used with an elevation difference greater than $\sigma_{\varphi}$. Starting from the assumption that there is no information on the target and that the Doppler adds very limited information to the classic sonar parameters, Doppler compression coefficients are not used in our localization algorithm. 


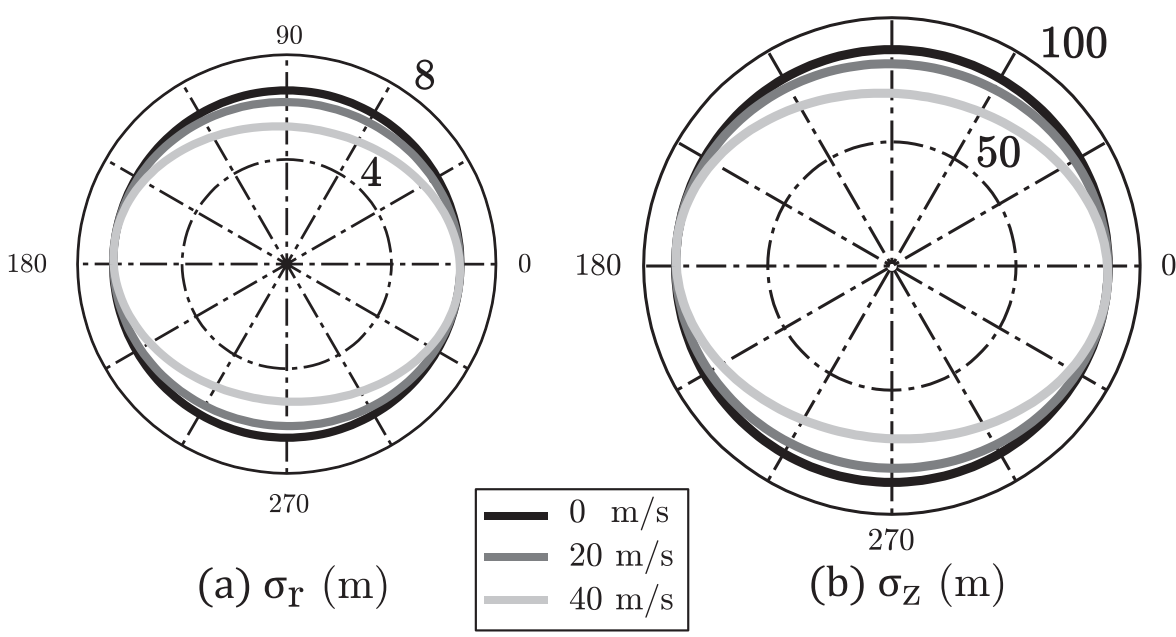

FIG. 7. Case $\mathrm{L}=2$. (a) Target-range standard deviation bound $\sigma_{r}$, and (b) target-depth standard deviation bound $\sigma_{z}$, as a function of the angle of the target-speed vector $\Phi_{t}$ for three different speeds of $\left\|\vec{V}_{t}\right\|=\left[\begin{array}{lll}0 & 10 & 20\end{array}\right] \mathrm{m} / \mathrm{s}$.

The probabilistic approach of the method is detailed here. The a posteriori probability density function (PDF) of the target position, $p\left(X \mid M^{m}\right)$, is the conditional PDF that the target is located at $X$ given the measurement vector $M^{m}$. Using the Bayes' rule, the a posteriori probability can be expressed as follows:

$$
p\left(X \mid M^{(m)}\right)=\frac{p\left(M^{(m)} \mid X\right) p(X)}{p\left(M^{(m)}\right)}
$$

where $p(X)$ and $p\left(M^{(m)}\right)$ are the PDFs of the target position and the measurements. In geo-acoustic inversion, the denominator $p\left(M^{(m)}\right)$, which is independent of $X$, is a constant. Replacing the denominator with a constant in the preceding equation gives

$$
p\left(X \mid M^{(m)}\right) \propto p\left(M^{(m)} \mid X\right) p(X) .
$$

For a given SSP, a target position $X$ can also be defined by the properties of eigenrays: $\left[\varphi^{e}(X), T^{e}(X)\right]$. So the conditional PDF, $p\left(M^{(m)} \mid X\right)$, can be re-written with the eigenray properties as follows:

$$
p\left(M^{(m)} \mid X\right)=p\left[\varphi^{(m)}, T^{(m)} \mid \varphi^{e}(X), T^{e}(X)\right],
$$

where $\varphi^{e}(X), T^{e}(X)$ are the angle and delay vectors of the eigenrays for the target position $X$. The number of eigenrays $E$ depends on the target position $X$. Assuming that variables $\varphi_{j}^{e}, T_{j}^{e}, \varphi_{i}^{(m)}, T_{i}^{(m)}$ for $1 \leq i \leq L$ and $1 \leq j \leq E(X)$ are all independent of each other, Eq. (28) becomes

$$
p\left(M^{(m)} \mid X\right)=\prod_{i, j}^{L, E(X)} p\left[\varphi_{i}^{(m)} \mid \varphi_{j}^{e}(X)\right] p\left[T_{i}^{(m)} \mid T_{j}^{e}(X)\right] .
$$

Considering that measurement errors follow a normal distribution:

$$
\begin{aligned}
& \varphi^{e}-\varphi^{(m)} \sim \mathcal{N}\left(0, \sigma_{\varphi}\right), \\
& T^{e}-T^{(m)} \sim \mathcal{N}\left(0, \sigma_{T}\right),
\end{aligned}
$$

where $\varphi^{e}$ and $T^{e}$ correspond to the eigenray properties between the sonar and the true target position, respectively. Finally the a posteriori PDF can be expressed as follows:

$$
\begin{aligned}
p\left(X \mid M^{(m)}\right) \propto & p(X) \prod_{i, j}^{L, E(X)} \frac{1}{2 \pi \sigma_{\varphi} \sigma_{T}} \\
& \times \exp \left(-\frac{\left[\varphi_{i}^{(m)}-\varphi_{j}^{e}(X)\right]^{2}}{2 \sigma_{\varphi}^{2}}-\frac{\left[T_{i}^{(m)}-T_{j}^{e}(X)\right]^{2}}{2 \sigma_{T}^{2}}\right)
\end{aligned}
$$

The target-position a priori $\mathrm{PDF}, p(X)$, is taken uniform in range and depth. The estimated target position is the maximum a posteriori of this a posteriori PDF. This maximization can be viewed as a minimization of the least squares on the elevations and delays. This a posteriori PDF can be interpreted as the product of $L 2-\mathrm{D}$ distributions or ellipses in the range-depth plane, in the same manner as detailed above for Fig. 3. To compute these PDFs, the one-way return travel times have to be estimated first.

\section{B. One-way return travel-time estimation}

The localization algorithm needs the one-way return travel times of the $L$ detections. In this paragraph, we explain how to choose these $L$ detections among $N$ detections, in order to compute $T$. Figure 8(a) illustrates four eigenrays for the sonar scenario described in Sec. II C, and Fig. 8(b) shows the ray elevations at the receiver for several detections as a function of the two-way travel time for two-way ray propagation. In this example, four eigenrays are considered between the sonar and the target: one down-refracted ray, one top-refracted ray, and two bottom-reflected rays. In Fig. 8(b), $N$ detections with sufficient SNR are represented by the points characterized by the elevation at the receiver, the two-way travel time, and the color that indicates the type of the one-way ray. To estimate the one-way return travel time of a point, the two-way path type has to be known. A twoway path is defined as "simple" if the acoustic wave took the same path going backward and forward, and in the opposite sense, a two-way path is defined as "combined" if the acoustic wave took different paths going backward and forward. So the one-way return travel time $T$ of a point is not necessarily half of the two-way travel time $\tau$. These simple or combined paths are indicated as either SP or CP for each 

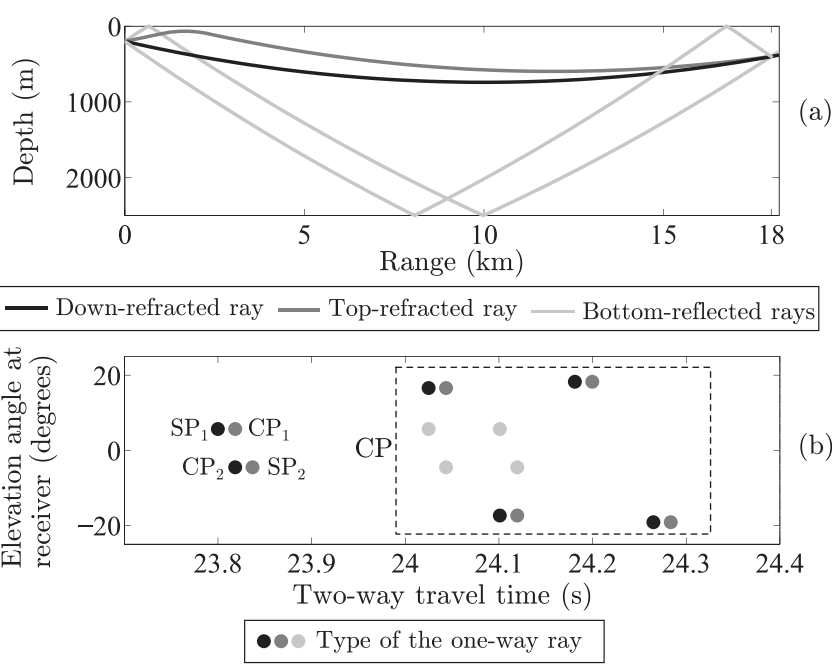

FIG. 8. (a) Example of four eigenrays between a sonar at $200 \mathrm{~m}$ in depth and a target at $400 \mathrm{~m}$ in depth and $18-\mathrm{km}$ range. (b) Elevation angles at the receiver as a function of the two-way travel time for the two-way ray propagation of the top panel. $\mathrm{SP}$, simple path; $\mathrm{CP}$, combined path. Example: $C P_{1}$ is composed of a top-refracted ray and a down-refracted ray; $S P_{2}$ is composed of two top-refracted rays.

detection to understand the path structure. As a first step, we assume that the detection with the shortest delay, $\tau_{1}$, came from a simple path. So the one-way return travel time of this first detection, $T_{1}$, can be computed as follows:

$$
T_{1}=\tau_{1} / 2
$$

Second, we assume that all of the detections that arrive first for each elevation angle at the receiver come from combined paths (except for the detection with the shortest delay). These come necessarily from the one-way path of the ray with the shortest one-way travel time. So the one-way return travel times of each of these detections can be computed as follows:

$$
T_{i}=\tau_{i}-T_{1}, \quad \text { for } \quad i=2, L .
$$

The variance of $T_{1}$ is $\sigma_{\tau_{1}}^{2}$. Noting that $T_{i}$ is obtained using a difference of independent and identically distributed random variables estimates, the variance of $T_{i}$ is $\sigma_{\tau_{1}}^{2}+\sigma_{\tau_{i}}^{2}$. This can be included in the estimator.

\section{Implementation}

The acoustic propagation simulator used was Bellhop, which is based on ray theory. ${ }^{20,21}$ This simulator allows the computation of the properties of eigenrays between an emitter and a receiver, given a SSP. For reasons of computation time, the eigenray properties are already computed on a large grid of target positions and on a given SSP. First, the target search grid has to be determined. In active sonar, the range search grid is easier to obtain, because the emission time is known. We assume that the target is between $r_{\min }$ and $r_{\text {max }}$ :

$$
\begin{aligned}
& r_{\min }=0.9 \frac{\tilde{c} T_{1}}{2}, \\
& r_{\max }=1.1 \frac{\tilde{c} T_{1}}{2},
\end{aligned}
$$

where $\tilde{c}=1500 \mathrm{~m} / \mathrm{s}$ is the mean sound speed. For the targetdepth search grid, we assume that the target-depth PDF is uniform between 0 and $1000 \mathrm{~m}$. Second, the a posteriori PDF is computed for one measurement pair $\left(\varphi_{i}^{m}, T_{i}^{m}\right)$ at a time on the defined search grid. For each position of the grid, an eigenray sort is carried out on the elevation angles and travel times. To increase the probability to have an intersection between the uncertainty ellipses, two scale factors, $k_{1}$ and $k_{2}$, are introduced, to expand the uncertainty margins. ${ }^{22}$ The uncertainties of the measurements can be re-written as follows:

$$
\begin{aligned}
& \sigma_{T}^{\prime}=k_{1} \times \sigma_{T}, \\
& \sigma_{\varphi}^{\prime}=k_{2} \times \sigma_{\varphi} .
\end{aligned}
$$

These scale factors are determined empirically and are often close to four to cover $99 \%$ of probabilities. Finally, the $a$ posteriori PDF is the product of each PDF computed for each measurement pair $\left(\varphi_{i}^{m}, T_{i}^{m}\right)$. The target position is then estimated by taking the maximum a posteriori of this PDF: i.e., the estimator that maximizes the PDF and minimizes the least squares. The existence of a solution is not guaranteed if there is no intersection between the ellipses. Compared to a local optimization method like gradient descend, the search grid method guarantees an absolute maximum in case of several local maxima.

\section{SIMULATION}

In this section, the localization algorithm is tested through two Monte-Carlo simulations. For these MonteCarlo simulations, three detections (i.e., top and downrefracted rays, and bottom-reflected ray) and 100 runs were used. The parameters of the active sonar scenario are the same as those used in Sec. II C. The first detection is carried out to estimate the bias and the variance of the estimator with respect to the CRBs. The elevation and time-delay measurements are random variables that follow a normal distribution around the true values of the elevations and time delays. The measurements given to the localization algorithm can be expressed as follows:

$$
\begin{aligned}
& \varphi_{i}^{m}=\varphi_{i}^{e}+\epsilon_{\varphi_{i}}, \\
& \tau_{i}^{m}=\tau_{i}^{e}+\epsilon_{\tau_{i}} \text { for } \quad i=1,3,
\end{aligned}
$$

where $\varphi_{i}^{e}$ and $\tau_{i}^{e}$ are the elevations and time delays for the true target position. Figure 9 shows the results for the first simulation. Figure 9(a) shows the a posteriori PDF for one draw. Figure 9(b) shows the two dimensional histogram of the Monte-Carlo simulation, where the dashed line represents the confidence ellipse at $95 \%$ of the CRBs. This simulation gives an expectation of the estimated target position of $399.8 \mathrm{~m}$ in depth and $18-\mathrm{km}$ range. This is slightly biased, but it appears to be caused by the finite size of the pixel or by the small number of Monte-Carlo runs. The estimated standard deviations are $1.92 \mathrm{~m}$ for the depth and $0.27 \mathrm{~m}$ for the range, which are small compared to the water-column height and close to the CRB standard deviations $\left(\sigma_{r}=0.35 \mathrm{~m}, \sigma_{z}=2 \mathrm{~m}\right)$. The estimated target positions are 


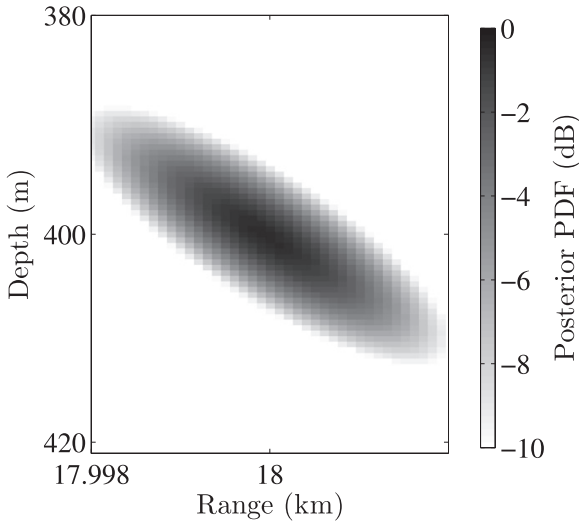

(a)

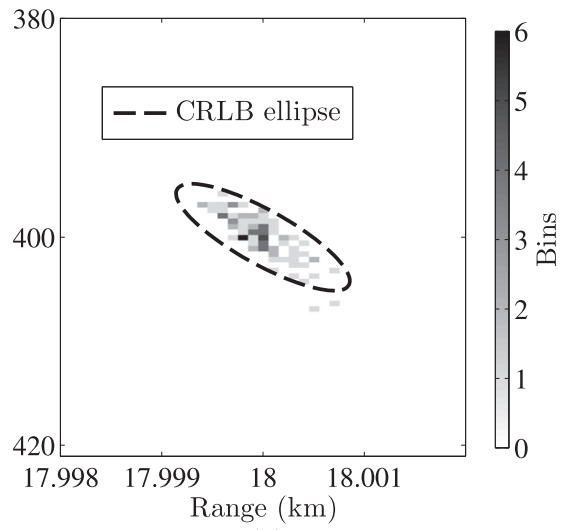

(b) almost in the $95 \%$ confidence ellipse, so this semi-active localization is quite efficient. The errors on the elevation angles and time-delay measurements are not preponderant compared to the errors of the environment variables. Errors in the sound-speed profile, sonar depth, bottom depth, and receiver tilt angle (environment inputs, generally) will probably cause larger bias and variance for the output variables. The robustness of the localization algorithm against SSP errors is then tested through the second Monte-Carlo simulation, by adding random SSPs to the previous simulation. First, the true elevations and time delays are perturbed by random SSPs, and second, the localization algorithm uses a bilinear approximation of the mean of the random SPPs. The measurements given to the localization algorithm can be expressed as follows:

$$
\varphi_{i}^{m}=\tilde{\varphi}_{i}^{e}+\epsilon_{\varphi_{i}},
$$

$$
\tau_{i}^{m}=\tilde{\tau}_{i}^{e}+\epsilon_{\tau_{i}} \quad \text { for } \quad i=1,3
$$

where $\varphi_{i}^{e}$ and $\tau_{i}^{e}$ are the true elevations and time delays, respectively, for a SSP that changes at every realization. The random SSP is generated by using empirical orthogonal functions (EOFs), ${ }^{23,24}$ These EOFs are extracted from an eigen decomposition of the covariance matrix from a database, which is a temporal historic of real SSPs for the months of July and August. The first six EOFs are shown in Figs. 10(a) and 10(b), and are used for the SSP generator, because $99 \%$ of the database can be represented by these six EOFs. The random part is performed by randomizing the EOF coefficients (i.e., eigenvalues) following a normal distribution. Figure 10(c) shows the mean SSP, the mean SSP with standard deviation, and the approximated SSP (bilinear) used for the localization algorithm. Figure 11 shows the results for the second simulation. Figure 11(a) shows the

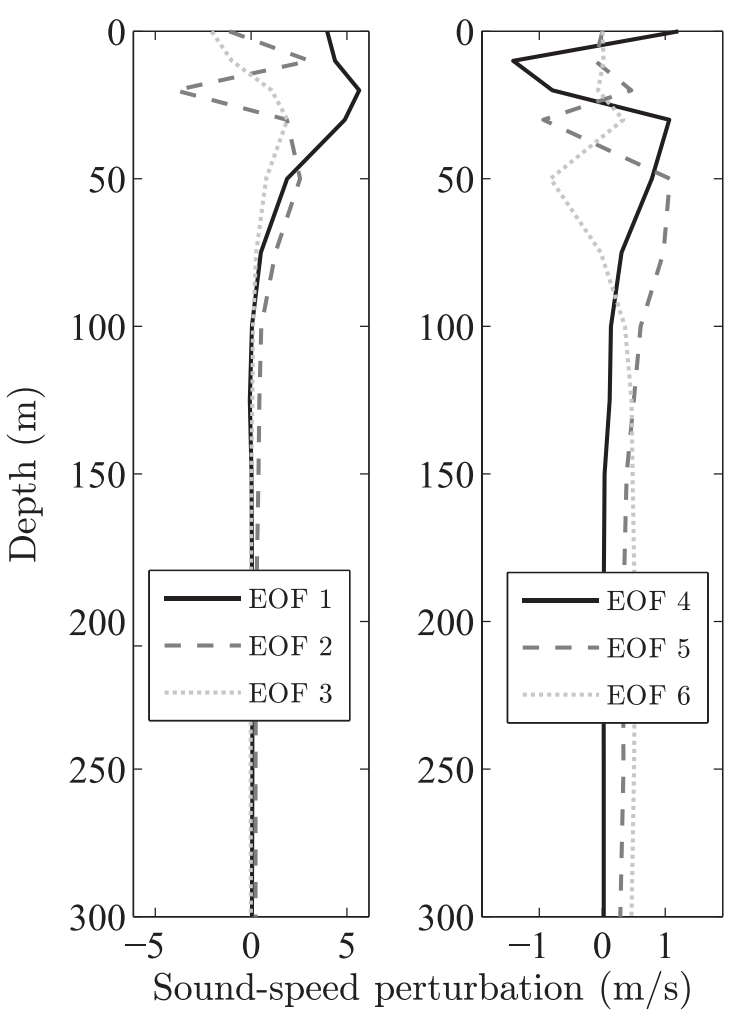

(a)

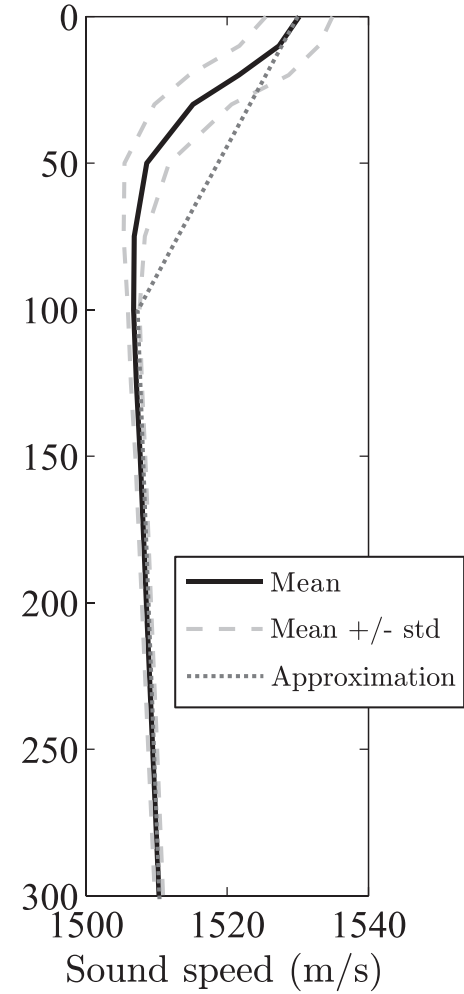

(c)
FIG. 10. (a), (b) The first six empirical orthogonal functions computed from the database (EOF 1-6). (c) The mean SSP with its standard deviation computed from the database, and the bilinear SSP approximation used by the localization algorithm: $\left(c_{1} ; z_{1}\right)=(0 \mathrm{~m}$; $1531 \mathrm{~m} / \mathrm{s}) ;\left(c_{2} ; z_{2}\right)=(100 \mathrm{~m} ; 1507 \mathrm{~m} / \mathrm{s})$; $\left(c_{3} ; z_{3}\right)=(2500 \mathrm{~m} ; 1546 \mathrm{~m} / \mathrm{s})$. 


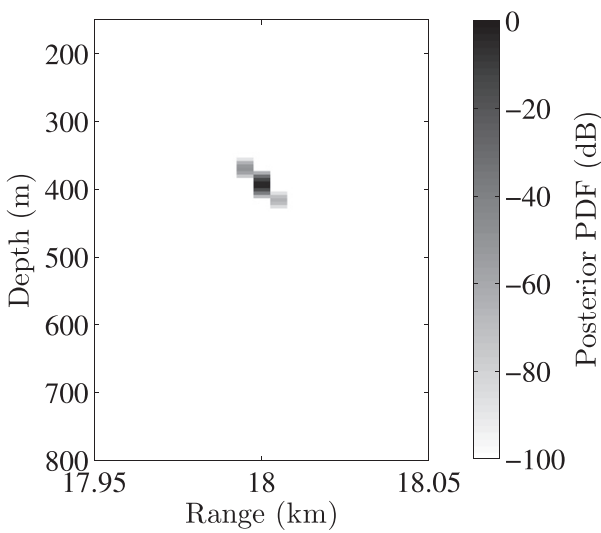

(a)

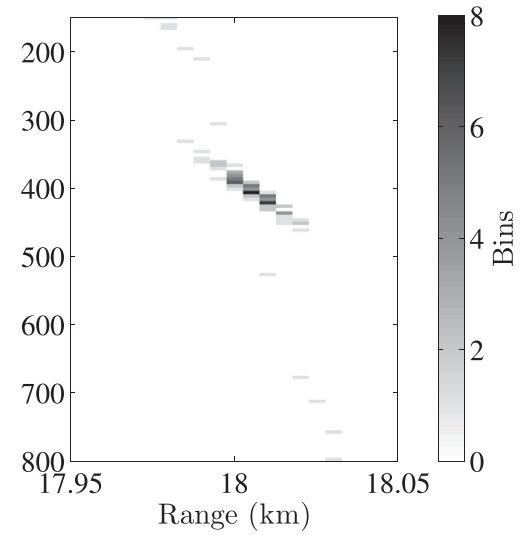

(b)
FIG. 11. (a) A posteriori PDF for one draw. (b) Two-dimensional histogram of the estimated target position, $\hat{z}_{t}$, of the Monte-Carlo simulation. The pixel intensity corresponds to the bin number. Statistical results: $\mathrm{E}\left[\hat{z}_{t}\right]=405 \mathrm{~m}$, $\sigma_{\hat{z}_{t}}=95 \mathrm{~m} ; \quad \mathrm{E}\left[\hat{r}_{t}\right]=18.005 \mathrm{~km}, \quad \sigma_{\hat{r_{t}}}$ $=10 \mathrm{~m}$. Configuration: $r=18 \mathrm{~km}, z$ $=400 \mathrm{~m}, \quad B=500 \mathrm{~Hz}, \quad \mathrm{SNR}=15 \mathrm{~dB}$, $\mathrm{k} 1=\mathrm{k} 2=4$, three detections used. a posteriori PDF for one draw. Figure 11(b) shows the two dimensional histogram of the Monte-Carlo simulation. The simulation gives an expectation of the estimated target position of $405 \mathrm{~m}$ in depth and $18.005-\mathrm{km}$ range, which is lowbiased but remains very acceptable. The estimated standard deviations are $95 \mathrm{~m}$ for the depth and $10 \mathrm{~m}$ for the range, which are not as good as the previous simulation, but are sufficient to approximately localize a target at depth regarding the water column height. However, the same simulation using two detections (i.e., top and down-refracted rays) provides a more biased expectation of the estimated target position and larger estimated standard deviations. This can be explained in terms of the top-refracted path, which is modified by the random SSPs, and therefore adds important bias and variance. The use of the third detection, which is a bottom-reflected ray, allows the localization algorithm to focus on the good target position, and therefore to give satisfying variance. The addition of a random SSP to the simulation shows that the target-position estimation and the associated standard deviation remain acceptable if the bottom-reflected ray is used. To go further, it would be interesting to simulate measurements that take into account random bottom depth, array depth, or array tilt. If the errors are important, the localization algorithm should include a priori the most important random parameter in the process.

\section{EXPERIMENTAL DATA}

This section proposes to validate our localization algorithm using experimental data from a real tank. An active sonar scenario in a shallow-water environment was reproduced in the experimental water tank of the ISTerre laboratory. ${ }^{25}$ The dimensions of the water tank were $1.9 \mathrm{~m}$ $\times 0.9 \mathrm{~m} \times 0.6 \mathrm{~m}$. A scaling factor of $200: 1$ was used to model a $5-\mathrm{kHz}$ active source in a deep ocean with a $0.7-\mathrm{m}$-diameter spherical target at a speed of $0.1 \mathrm{~m} / \mathrm{s}$. At a real scale, the metallic target would be immersed at $5.4 \mathrm{~m}$ depth, and the first hydrophone of the vertical uniform linear array would be at a depth of $4.8 \mathrm{~m}$. The uniform linear array would be $112 \mathrm{~m}$ away from the target when the waveform is emitted. At the laboratory scale, the uniform linear array was composed of 64 half-wavelength-spaced transducers that had a $1-\mathrm{MHz}$ carrier frequency and a $1-\mathrm{MHz}$ bandwidth at $-6 \mathrm{~dB}$, with a sampling frequency of $F_{e}=20 \mathrm{MHz}$. The target was a $3.5-\mathrm{mm}$ spherical lead that was hung from a motorized arm. High waves of a few millimeters were generated on the surface layer to add random perturbation to the propagation. The experimental setup is illustrated in Fig. 12. The motorized arm was used to set the target accurately in position and to move it horizontally. The target was $230 \mathrm{~mm}$ away from the transducer array at time $t=0$. The target was then shifted to the left at $550 \mathrm{~mm}$ at a constant horizontal velocity of $0.1 \mathrm{~m} /$ $\mathrm{s}$, as shown on Fig. 13. The emission of the waveform was realized with the transducer located in the middle of the uniform linear array, to create a spherical wave that propagated at all elevation angles with a small azimuth beam width. The waveform was a binary phase-shift keying signal with a bandwidth of $600 \mathrm{kHz}$ and a time duration of $500 \mu \mathrm{s}$. In this example, the elevation angle and the time resolution are $\varphi_{-3 \mathrm{~dB}}=0.8 \mathrm{deg}$ and $\tau_{-3 \mathrm{~dB}}=0.7 \mu \mathrm{s}$, respectively, the SSP was constant, and the number of rays detected was only two (i.e., direct and surface-reflected rays). Figure 13 shows the a posteriori PDF for one draw. The dashed lines represent the uncertainty ellipses for the direct and surface-reflected rays. The two ellipses are not well focused, and only their borders intersect. The true target depth was $z_{t}=37 \mathrm{~mm} \pm 2 \mathrm{~mm}$ (at the real scale: $z_{t}=5.4 \mathrm{~m} \pm 0.4 \mathrm{~m}$ ) and the localization method provides an expectation of the estimated target depth of $\mathrm{E}\left[\hat{z}_{t}\right]=31.3 \mathrm{~mm} \pm 1.5 \mathrm{~mm}$ (at the real scale: $\mathrm{E}\left[\hat{z}_{t}\right]=6.3 \mathrm{~m}$ $\pm 0.3 \mathrm{~m}$ ) over 10 realizations. The statistics of the targetdepth estimation could be biased by the array tilt, the surfacereflected ray, which suffers from the surface perturbations, and the low number of realizations. The target-depth estimator is biased of $1 \mathrm{~m}$ at the real scale, so this validate our localization method with these experimental tank data.

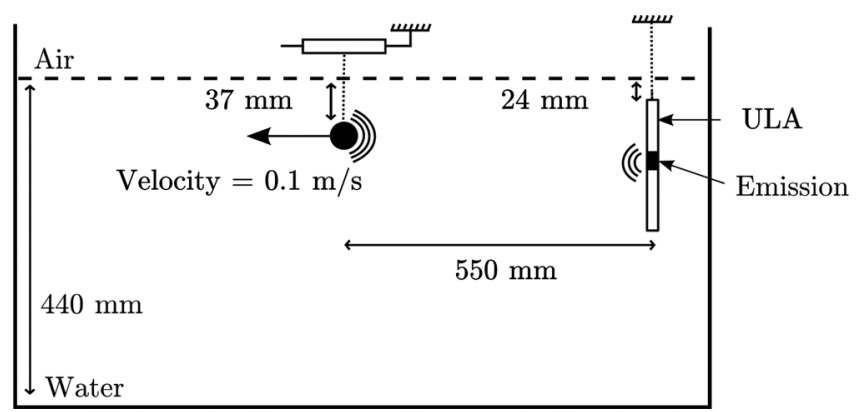

FIG. 12. Setup of the experimental water-filled tank. The experiment was composed of a vertical uniform linear array (ULA) with 64 transducers, and a moving spherical target. A waveform was emitted by the 33rd transducer when the target had reached the constant speed of $V=0.1 \mathrm{~m} / \mathrm{s}$. 


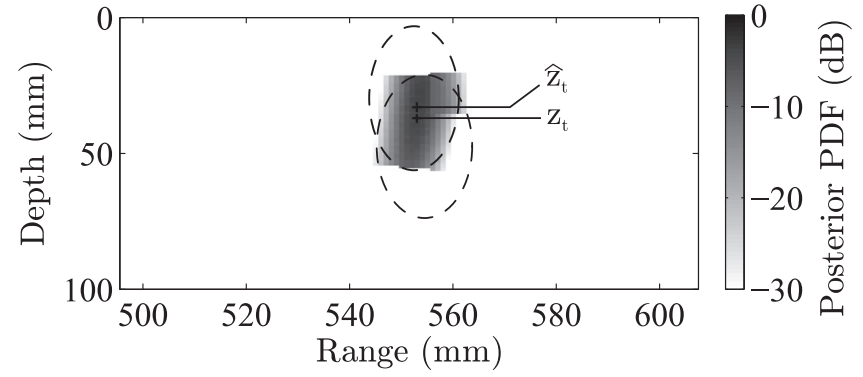

FIG. 13. Real data. A posteriori PDF for one draw. Estimated target position: $\hat{z_{t}}=31.3 \mathrm{~mm}$ (true target position: $z_{t}=37 \mathrm{~mm} \pm 2 \mathrm{~mm}$ ). The dashed lines represent the uncertainty ellipses for the direct and surface-reflected rays. $\mathrm{k} 1=\mathrm{k} 2=4$.

\section{CONCLUSIONS}

Research in the target localization domain with an active sonar remains a poorly discussed topic. This paper focuses on estimation of the depth of a target using elevations and timedelay measurements in active sonar and deep water, and especially for a summer Mediterranean SSP. A new targetlocalization approach in active sonar and new CRBs for target localization for depth and range are proposed here.

These new CRBs were proposed for a bilinear SSP in a vertical plane in order to estimate the variance of the targetdepth estimate. We have shown that an increase in the number of acoustic paths used decreases the uncertainty of the target position. The CRB results suggest that target-depth estimation is possible with low uncertainty compared to the water-column height for this active sonar scenario. The semi-active localization based on ray back-propagation and a probabilistic approach was tested for Monte-Carlo simulations and for water-tank experimental data. This method is also discussed in terms of ray identification and how the combined acoustic paths were managed. In others words, rays that have a different path from sonar to target and from target to array are taken into account. The simulation results suggest that the estimator is near the CRBs and that targetdepth estimation is possible with low uncertainty compared to the water-column height for a target at $400 \mathrm{~m}$ in depth and 18-km range. However, some environmental parameters can increase the bias and the variance of the target-depth estimator, such as random bottom depth, array depth, or array tilt. The results from the experimental data with surface noise reveal good estimation of the target depth and validate the localization algorithm for a constant SSP.

\section{ACKNOWLEDGMENTS}

The author wishes to thank Philippe Roux for providing access and help to the experimental water tank of the ISTerre laboratory, ${ }^{25}$ and Xavier Cristol for many fruitful discussions.

\section{APPENDIX A: EIGENRAYS IN A BILINEAR SOUND-SPEED PROFILE}

We detail here the analytic expression of the functions $\mathrm{G}$ and $\mathrm{H}$, which were introduced by Baer and Jacobson ${ }^{16}$ and rewritten later more compactly by Jauffret and Blanc-Benon. ${ }^{12}$ This function system describes the coordinates of any type of ray in a bilinear SSP, and these are correct assuming that $c_{1}>c_{3}>c_{2}$, otherwise the bottom and the surface have to be exchanged, and the $z$ axis has to be positive downwards

$$
\begin{aligned}
G(\varphi, z)= & \frac{c_{0}}{2 \cos \varphi}\left\{\frac{1}{g_{1}}[(-1-\alpha) \sin \varphi+(1+\beta) \sin \Phi\right. \\
& \left.-2 \nu(2 P-\gamma+\delta) \sin \varphi_{1}\right]-\frac{1}{g_{2}}[(1-\alpha) \sin \varphi \\
& \left.+(\beta-1) \sin \Phi-4 \mu P \sin \varphi_{3}\right] \\
& \left.+\left(\frac{1}{g_{1}}-\frac{1}{g_{2}}\right)(4 P-2+\sigma) \sin \varphi_{2}\right\}, \\
H(\varphi, z)= & \frac{1}{g_{1}} \ln \left[\Psi(\varphi)^{-(1+\alpha) / 2} \Psi(\Phi)^{(1+\beta) / 2}\right. \\
& \left.\times \Psi\left(\varphi_{1}\right)^{-\nu(2 P-\gamma+\delta)}\right]-\frac{1}{g_{2}} \ln \left[\Psi(\varphi)^{(1-\alpha) / 2}\right. \\
& \left.\times \Psi(\Phi)^{(\beta-1) / 2} \Psi\left(\varphi_{3}\right)^{-2 \mu P}\right] \\
& +\left(\frac{1}{g_{1}}-\frac{1}{g_{2}}\right) \ln \left[\Psi\left(\varphi_{2}\right)^{2 P-1+\sigma / 2}\right],
\end{aligned}
$$

where $g_{1}$ and $g_{2}$ are the algebraic gradients of celerity in the surface layer and the deep layer, respectively, and $P$ is the number of bottom reflections or refractions. The minor parameters can be expressed as follows:

$$
\begin{aligned}
& g_{1}=\frac{c_{1}-c_{2}}{z_{2}}, \quad g_{2}=-\frac{c_{3}-c_{2}}{z_{3}-z_{2}}, \\
& c(z)=c_{2}+\left(B g_{1}-D g_{2}\right)\left(z-z_{2}\right), \\
& \sin \Phi=\delta \frac{c}{c_{0}} \sqrt{\frac{c_{0}}{c}-1+\sin ^{2} \varphi}, \\
& \sin \varphi_{i}=\frac{c_{i}}{c_{0}} \sqrt{\frac{c_{0}^{2}}{c_{i}^{2}}-1+\sin ^{2} \varphi}, \\
& \Psi(\varphi)=\tan (\varphi / 2+\pi / 4), \\
& \alpha= \begin{cases}+1 & \text { if } z<z_{2} \\
-1 & \text { if } z>z_{2},\end{cases} \\
& \beta= \begin{cases}+1 & \text { if } z_{0}<z_{2} \\
-1 & \text { if } z_{0}>z_{2},\end{cases} \\
& \gamma=\operatorname{sign}(\varphi), \quad \delta=\operatorname{sign}(\Phi), \\
& \sigma=|\alpha+\gamma|+|\beta-\delta|-\gamma+\delta, \\
& \mu=\nu=0 \text { if } \operatorname{SOFAR} \text { rays }, \\
& \mu=1, \nu=0 \text { if RBR rays, } \\
& \mu=0, \nu=1 \text { if RSR rays, } \\
& \mu=\nu=1 \text { if SRBR rays, }
\end{aligned}
$$

where $c(z)$ (respectively, $c_{0}$ ) is the sound speed at the target depth $\mathrm{z}$ (respectively, the sonar depth $z_{0}$ ). The other parameters control the type of eigenray, such as SOFAR, refracted/bottom-reflected, refracted/surface-reflected and surfacereflected/bottom-reflected. The equations of $\alpha, \beta$ (exchange signs $<$ and $>$ ) and $\sin \mu_{i}$, $\sin \mu_{0}$ used by Blanc-Benon ${ }^{12}$ were typing errors, and these are corrected here through reading of the original paper. ${ }^{16}$ 


\section{APPENDIX B: PARTIAL DERIVATIVES OF G AND $H$}

The partial derivatives of $G$ and $H$ can be expressed as follows:

$$
\begin{aligned}
\frac{\partial G}{\partial z}= & -\frac{c \cos \varphi}{c_{0} \sin \Phi}\left(\frac{C}{g_{1}}-\frac{E}{g_{2}}\right)\left(B g_{1}-D g_{2}\right), \\
\frac{\partial G}{\partial \varphi}= & \frac{c_{0} \sin \varphi}{2 \cos ^{2} \varphi}\left[\frac{1}{g_{1}}\left(\frac{2 B}{\sin \varphi}+\frac{2 C}{\sin \Phi}-\frac{2 A}{\sin \varphi_{1}}\right)-\frac{1}{g_{2}}\left(\frac{2 D}{\sin \varphi}+\frac{2 E}{\sin \Phi}-\frac{4 \mu P}{\sin \varphi_{3}}\right)+\left(\frac{1}{g_{1}}-\frac{1}{g_{2}}\right)\left(\frac{1}{\sin \varphi_{2}}\right)\right], \\
\frac{\partial H}{\partial z}= & -\left(\frac{C}{g_{1}}-\frac{E}{g_{2}}\right)\left(B g_{1}-D g_{2}\right) \frac{c \cos ^{2} \varphi\left(\Psi^{2}(\Phi)+1\right)}{2 c_{0}^{2} \Psi(\Phi)|\cos \Phi| \sin \Phi}, \\
\frac{\partial H}{\partial \varphi}= & \frac{1}{g_{1}}\left\{\frac{A c_{1}^{2} \sin 2 \varphi\left[\Psi^{2}\left(\varphi_{1}\right)+1\right]}{4 c_{0}^{2} \Psi\left(\varphi_{1}\right)\left|\cos \varphi_{1}\right| \sin \varphi_{1}}+\frac{C c^{2} \delta^{2} \sin 2 \varphi\left[\Psi^{2}(\varphi)+1\right]}{4 c_{0}^{2} \Psi(\Phi)|\cos \Phi| \sin \Phi}+\frac{B\left[\Psi^{2}(\varphi)+1\right]}{2 \Psi(\varphi)}\right\} \\
& -\frac{1}{g_{2}}\left\{-\frac{c_{3}^{2} P \mu \sin 2 \varphi\left[\Psi^{2}\left(\varphi_{3}\right)+1\right]}{4 c_{0}^{2} \Psi\left(\varphi_{3}\right)\left|\cos \varphi_{3}\right| \sin \varphi_{3}}+\frac{E c^{2} \delta^{2} \sin 2 \varphi\left[\Psi^{2}(\Phi)+1\right]}{4 c_{0}^{2} \Psi(\Phi)|\cos \Phi| \sin \Phi}+\frac{D\left[\Psi^{2}(\varphi)+1\right]}{2 \Psi(\varphi)}\right\} \\
& +\left(\frac{1}{g_{1}}-\frac{1}{g_{2}}\right) \frac{c_{2}^{2}(2 P+\sigma / 2-1) \sin 2 \varphi\left[\Psi^{2}\left(\varphi_{2}\right)+1\right]}{4 c_{0}^{2} \Psi\left(\varphi_{2}\right)\left|\cos \varphi_{2}\right| \sin \varphi_{2}},
\end{aligned}
$$

where A, B, C, and D are intermediate variables, and can be expressed as follows:

$$
\begin{array}{ll}
A=\nu(2 P+\delta-\gamma), & B=\frac{-\alpha-1}{2}, \quad C=\frac{\beta+1}{2}, \\
D=\frac{1-\alpha}{2}, & E=\frac{\beta-1}{2} .
\end{array}
$$

${ }^{1}$ M. Meyer and J.-P. Hermand, "Backpropagation techniques in ocean acoustic inversion: Time reversal, retrogation and adjoint model-A review," in Acoustic Sensing Techniques for the Shallow Water Environment (Springer, the Netherlands, 2006), pp. 29-46.

${ }^{2}$ A. B. Baggeroer, W. A. Kuperman, and P. N. Mikhalevsky, "An overview of matched field methods in ocean acoustics," IEEE J. Ocean. Eng. 18, 401-424 (1993).

${ }^{3}$ A. Richardson and L. Nolte, "A posteriori probability source localization in an uncertain sound speed, deep ocean environment," J. Acoust. Soc. Am. 89, 2280-2284 (1991).

${ }^{4}$ S. E. Dosso and M. J. Wilmut, "Comparison of focalization and marginalization for Bayesian tracking in an uncertain ocean environment," J. Acoust. Soc. Am. 125, 717-722 (2009).

${ }^{5}$ S. E. Dosso and M. J. Wilmut, "Bayesian focalization: Quantifying source localization with environmental uncertainty," J. Acoust. Soc. Am. 121, 2567-2574 (2007).

${ }^{6}$ M. D. Collins and W. Kuperman, "Focalization: Environmental focusing and source localization,” J. Acoust. Soc. Am. 90, 1410-1422 (1991).

${ }^{7} \mathrm{P}$. Voltz and I. Lu, "A time-domain backpropagating ray technique for source localization," J. Acoust. Soc. Am. 95, 805-812 (1994).

${ }^{8}$ I.-T. Lu, "Simultaneous characterization of source, array and environment using a ray travel-time inversion approach," J. Comput. Acoust. 5, 193-218 (1997).

${ }^{9}$ I.-T. Lu, "Robustness of a ray travel-time inversion approach," J. Acoust. Soc. Am. 106, 2442-2453 (1999).

${ }^{10}$ G. Hickman and J. L. Krolik, "Matched-field depth estimation for active sonar," J. Acoust. Soc. Am. 115, 620-629 (2004).
${ }^{11} \mathrm{~K}$. T. Hjelmervik, "Target depth estimation using a ray backpropagation scheme on mid-frequency active sonar data," in Proceedings of the European Conference on Underwater Acoustics (2010).

${ }^{12} \mathrm{P}$. Blanc-Benon and C. Jauffret, "TMA from bearings and multipath time delays," IEEE Trans. Aerospace Electron. Syst. 33, 813-824 (1997).

${ }^{13}$ S. M. Kay, Fundamentals of Statistical Signal Processing, Volume I: Estimation Theory (Prentice-Hall, Englewood Cliffs, NJ, 1993), pp. 45, 27-77.

${ }^{14}$ H. L. Van Trees, Detection, Estimation, and Modulation Theory, Optimum Array Processing (Wiley, New York, 2004), pp. 925-927.

${ }^{15}$ W. S. Burdic, Underwater Acoustic Signal Analysis (Prentice-Hall, Englewood Cliffs, NJ, 1984), pp. 114-121, 328, 406.

${ }^{16} \mathrm{R}$. Baer and M. Jacobson, "Sound transmission in a channel with bilinear sound speed and environmental variations," J. Acoust. Soc. Am. 54, 80-91 (1973).

${ }^{17}$ H. L. Van Trees, Detection, Estimation, and Modulation Theory, RadarSonar Processing and Gaussian Signals in Noise (Wiley, New York, 2004), pp. 238-241, 300.

${ }^{18}$ J.-P. Hermand and W. I. Roderick, "Delay-Doppler resolution performance of large time-bandwidth-product linear FM signals in a multipath ocean environment," J. Acoust. Soc. Am. 84, 1709-1727 (1988).

${ }^{19} \mathrm{G}$. Robertson, "Operating characteristics for a linear detector of $\mathrm{cw}$ in narrow-band Gaussian noise," Bell Syst. Tech. J. 46, 755-774 (1967).

${ }^{20}$ M. B. Porter, "Bellhop Gaussian beam/finite element beam code," Available in the Acoustics Toolbox, http://oalib.hlsresearch.com/Rays (Last viewed 1/9/2016).

${ }^{21}$ M. B. Porter, "The bellhop manual and user?s guide: Preliminary draft," Heat, Light, and Sound Research, Inc., La Jolla, CA., USA, Tech. Rep. (2011).

${ }^{22}$ S. C. Stiros and V. Saltogianni, "Solution of underdetermined systems of equations with gridded a priori constraints," SpringerPlus 3, 1-15 (2014).

${ }^{23}$ L. R. LeBlanc and F. H. Middleton, "An underwater acoustic sound velocity data model," J. Acoust. Soc. Am. 67, 2055-2062 (1980).

${ }^{24} \mathrm{~W}$. Xu and H. Schmidt, "System-orthogonal functions for sound speed profile perturbation,” IEEE J. Ocean. Eng. 31, 156-169 (2006).

${ }^{25} \mathrm{P}$. Roux, I. Iturbe, B. Nicolas, J. Virieux, and J. I. Mars, "Travel-time tomography in shallow water: Experimental demonstration at an ultrasonic scale," J. Acoust. Soc. Am. 130, 1232-1241 (2011). 